\title{
Scalable Wavelength-Converting Crossbar Switches
}

\author{
Hilmi Volkan Demir, Vijit A. Sabnis, Student Member, IEEE, Jun-Fei Zheng, Onur Fidaner, Student Member, IEEE, \\ James S. Harris, Jr., Fellow, IEEE, and David A. B. Miller, Fellow, IEEE
}

\begin{abstract}
We report scalable low-power wavelength-converting crossbar switches that monolithically integrate two-dimensional compact arrays of surface-normal photodiodes with quantum-well waveguide modulators. We demonstrate proof-of-concept, electrically reconfigurable $2 \times 2$ crossbars that perform unconstrained wavelength conversion across $35 \mathrm{~nm}$ in the $C$-band (1530-1565 nm), using only $<4.3-\mathrm{mW}$ absorbed input optical power, and with 10-dB extinction ratio at $1.25 \mathrm{~Gb} / \mathrm{s}$. Such wavelength-converting crossbars provide complete flexibility to selectively convert any of the input wavelengths to any of the output wavelengths at high data bit rates in telecommunication, with the input and output wavelengths being arbitrarily chosen within the $C$-band.
\end{abstract}

Index Terms-Electroabsorption, integrated optoelectronics, optical frequency conversion, optoelectronic devices, quantum-well devices.

\section{INTRODUCTION}

A LL-OPTICAL wavelength conversion has been extensively investigated, and different wavelength converters and optical switches have been demonstrated with promising results [1]-[3]. These wavelength converters have, however, previously not been scaled into compact two-dimensional arrays to realize chip-scale reconfigurable multichannel wavelength conversion systems. For instance, an integrated wavelength-converting optical crossbar switch that allows for arbitrary mapping of the input wavelengths to the output wavelengths has not been implemented with these prior approaches. To address this problem, we introduce a novel scalable wavelength-converting optical crossbar switch that consists of a two-dimensional array of optically controlled optoelectronic switches placed at its cross nodes [4].

\section{Device Structure AND OPERATION}

Fig. 1 shows a plan view of a fabricated $2 \times 2$ wavelengthconverting crossbar with a total of four optoelectronic switch elements. Each switch in the crossbar includes an InGaAsP-InP quantum-well waveguide modulator and a surface-illuminated InGaAs photodiode as a part of its optoelectronic circuit (as

Manuscript received November 24, 2003; revised June 3, 2004. This work was supported by Intel Corporation, and by PTAP (funded by NSF and DARPA).

H. V. Demir was with the Edward L. Ginzton Laboratory and Solid State and Photonics Laboratory, Stanford University, Stanford, CA 94305 USA. He is now with the Nanotechnology Research Center, Bilkent University, Ankara 06800, Turkey (e-mail: volkan@fen.bilkent.edu.tr).

V. A. Sabnis, O. Fidaner, J. S. Harris, and D. A. B. Miller are with the Edward L. Ginzton Laboratory and Solid State and Photonics Laboratory, Stanford University, Stanford, CA 94305 USA.

J. F. Zheng is with Intel Strategic Technology, Intel Corporation, Santa Clara, CA 95052 USA.

Digital Object Identifier 10.1109/LPT.2004.834473

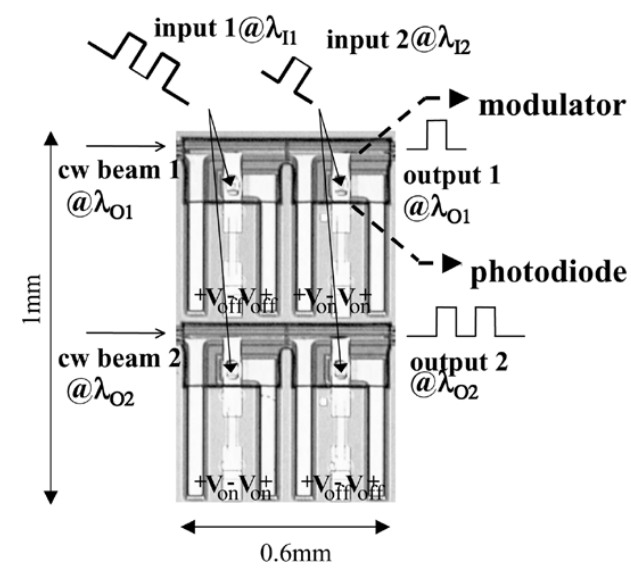

Fig. 1. Plan-view microscope picture of a $2 \times 2$ wavelength-converting crossbar. Each optical input channel is transferred to one of the output channels of our choice while concurrently converting its wavelength.

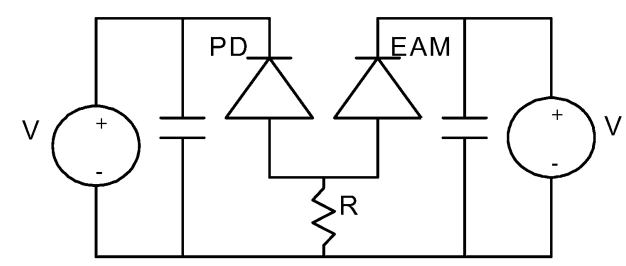

Fig. 2. Simplified circuit model. The photocurrent from the photodiode (PD) generated a voltage drop across the resistor $(\mathrm{R})$, screening the voltage across the electroabsorption modulator (EAM).

shown in Fig. 2) on an InP substrate [5]. The photodiodes and modulators of the switches are monolithically integrated into a compact circuit area, using a selective area regrowth technique [6].

The function of these switches is to transfer optical signals from the input data streams, incident on their respective photodiodes, onto the continuous-wave beams coupled into their modulator waveguides, provided that the photodiodes and modulators are properly reverse-biased. When slightly forward-biased, the photodiodes will not extract photocurrent and the modulators will be transparent. Therefore, each of these switches located at the cross nodes can be independently enabled and disabled to reconfigure the crossbar as desired by simply changing their biases accordingly.

The integrated broad-band absorbing InGaAs photodiodes detect over the entire telecommunication wavelength range and the InGaAsP quantum-well modulators are designed to modulate optical transmission over the telecommunication center $(C$-) band $(1535-1565 \mathrm{~nm})$ with appropriate reverse biasing. Consequently, these optically controlled optoelectronic switches provide unconstrained wavelength conversion across the entire $C$-band [4], [7]. 

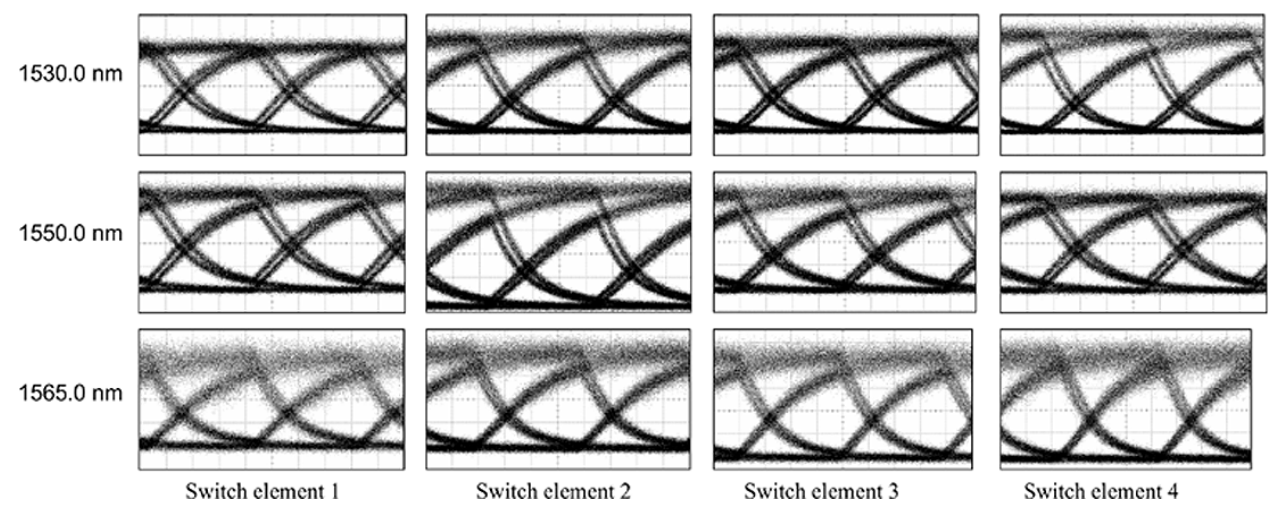

Switch element 4

Fig. 3. Eye diagrams from four switch elements in a $2 \times 2$ array for NRZ operation. Output wavelengths are at 1530.0, 1550.0, and 1565.0 nm. All measurements are taken at $1.25 \mathrm{~Gb} / \mathrm{s}$, with the input wavelength at $1551.7 \mathrm{~nm}$. (Horizontal: $200 \mathrm{ps} / \mathrm{div}$. Vertical: $\sim 333 \mathrm{~mW} / \mathrm{div}$ ).

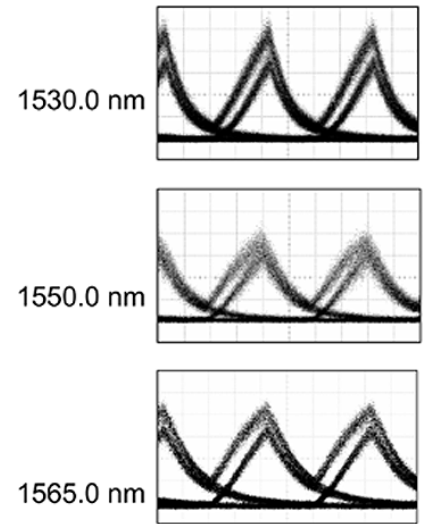

Switch element 1
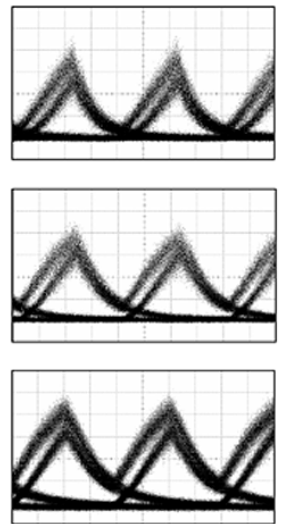

Switch element 2
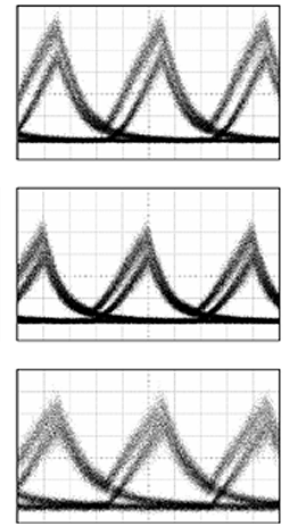

Switch element 3
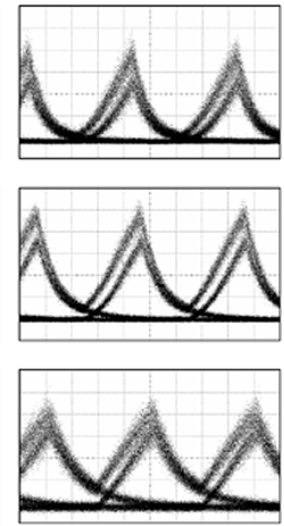

Switch element 4

Fig. 4. Eye diagrams from four switch elements in a $2 \times 2$ array for RZ operation. Output wavelengths are at 1530.0, 1550.0, and 1565.0 nm. All measurements are taken at $1.25 \mathrm{~Gb} / \mathrm{s}$, with the input wavelength at $1551.7 \mathrm{~nm}$. (Horizontal: $200 \mathrm{ps} / \mathrm{div}$. Vertical: $333 \mathrm{~mW} / \mathrm{div}$ ).

A theoretical device model that includes carrier transport, on-chip integrated optoelectronic circuit, and off-chip biasing circuit confirms high-speed wavelength conversion at data bit rates important for telecommunication, for example at $10 \mathrm{~Gb} / \mathrm{s}$ using a lumped modulator [7], and at even higher speeds using a traveling-wave modulator [3]. Our previous proof-of-principle experimental results also demonstrate efficient wavelength conversion using single devices up to $2.5 \mathrm{~Gb} / \mathrm{s}$ with milliwatt-level optical power for $>10-\mathrm{dB}$ extinction ratios across $45 \mathrm{~nm}$ covering the $C$-band in nonreturn-to-zero (NRZ) and return-to-zero (RZ) schemes [4].

The $2 \times 2$ array depicted in Fig. 1 includes two side-by-side waveguides, each incorporating two switches. For crossbar operation, with the use of a diffractive spot generator or fiber splitters, the optical Input 1 at $\lambda_{I 1}$ could be optically distributed to the two leftmost photodiodes, each connecting to a different waveguide, and similarly, the optical Input 2 at $\lambda_{I 2}$ could be distributed to the rightmost photodiodes. Two continuous-wave beams at $\lambda_{O 1}$ and $\lambda_{O 2}$ could also be separately coupled into the two waveguides. By electrically enabling the bottom left wavelength converter and the top right one, and disabling the rest of them, the crossbar can be configured to transfer optical information from Input 1 to Output 2 and from Input 2 to Output 1 , with the selected wavelength mapping of $\lambda_{I 1} \rightarrow \lambda_{O 2}$ and $\lambda_{I 2} \rightarrow \lambda_{O 1}$, as shown in Fig. 1. Similarly, the complementary mapping of $\lambda_{I 1} \rightarrow \lambda_{O 1}$ and $\lambda_{I 2} \rightarrow \lambda_{O 2}$ is possible through electrically enabling the top left wavelength converter and the bottom right one, and disabling the rest.

Fig. 2 shows a simplified circuit diagram of a single wavelength converter. An on-chip thin-film resistor $(\mathrm{R})$ interconnects p-regions of the photodiode and the modulator to a common ground. The photodiode and the modulator are separately reverse-biased using a pair of bypass capacitors connected to dc power supplies. When the input data stream at $\lambda_{I}$ is incident on the photodiode, the resulting photocurrent modulates the voltage across the local resistor and the modulator, which in turn modulates its output transmission at $\lambda_{O}$, for wavelength conversion from $\lambda_{I} \rightarrow \lambda_{O}$. With the tight integration of the photodiode and the modulator, and the use of bypass capacitors, the photogenerated high-speed signals are localized within the lumped circuit elements of the switch. Thus, the switching bandwidth is set predominantly by the total internal device resistance-capacitance $(R C)$ time constant. This allows for low-power high-speed operation without requiring conventional high-speed electrical signal propagation or impedance matching.

Because of the tradeoff between the switching speed and optically induced voltage swing, the device $R C$ is optimized for high extinction ratio given the operation speed. For $10-\mathrm{Gb} / \mathrm{s}$ operation, the wavelength converter design includes a $30 \times 30 \mu \mathrm{m}$ photodiode with a $1.46-\mu \mathrm{m}$-thick $i$-region and a $2-\mu \mathrm{m}$-wide $300-\mu \mathrm{m}$-long modulator with a $0.37-\mu \mathrm{m}$-thick $i$-region, and a 
resistor of $120 \Omega$. For the purpose of preliminary experimental demonstration at $1.25 \mathrm{~Gb} / \mathrm{s}$, we fabricated the $2 \times 2$ crossbar shown in Fig. 1 with a 1.5 - $\mu \mathrm{m}$-thick $i$-region in the photodiode, a 0.5 - $\mu \mathrm{m}$-thick $i$-region in the modulator with a width of $3 \mu \mathrm{m}$, and an on-chip TaN thin film resistor of $650 \Omega$.

\section{EXPERIMENTAL RESULTS}

We report proof-of-concept $C$-band wavelength conversion results with $10-\mathrm{dB}$ extinction ratios from a $2 \times 2$ array with four switch elements. Fig. 3 shows NRZ eye diagrams taken separately from each of the four switch elements at three output wavelengths of $1530.0,1550.0$, and $1565.0 \mathrm{~nm}$ at $1.25 \mathrm{~Gb} / \mathrm{s}$ using an optically preamplified receiver to observe the output signal. Fig. 4 shows a similar set of data for RZ operation. To enable optical switching, the photodiode and the modulator of a wavelength converter under test were reverse-biased at $20.0 \mathrm{~V}$, and at 3.7-7.0 $\mathrm{V}$ depending on the operating output wavelength, respectively. The other wavelength converter along the same waveguide was disabled by slightly forward biasing its unused photodiode and modulator. This prevented this photodiode from extracting photocurrent and inducing a voltage swing across the connecting modulator, and forced the modulator quantum wells into the transparent region removing the background quantum-well absorption. In all cases, the input wavelength was $1551.7 \mathrm{~nm}$ and the absorbed optical power in the input beam was $<4.3 \mathrm{~mW}$. With the input beam photogenerating $\sim 3 \mathrm{~mA}$ of current, we observed an optically induced $\sim 3.9-\mathrm{V} / \mu \mathrm{m}$ field swing, which is comparable to that required by conventional electroabsorption modulators to achieve $>10-\mathrm{dB}$ extinction ratios. In these experiments, we observed power fluctuations in the output beams coupled out of the waveguides due to mechanical vibrations and sensitivity of the waveguide alignment to vibrations. During optical switching, such amplitude fluctuations further gave rise to extra timing jitter in the output eye diagrams, although there is no physical mechanism inherent to the operation of our device that causes additional jitter.

We also conducted crosstalk experiments for every possible combination in the $2 \times 2$ array with two of the wavelength converters along two different waveguides oN (enabled) and the others OFF (disabled). There was no noticeable crosstalk between different switch nodes due to a possible electrical or optical coupling.

In an $n \times n$ crossbar operation, since each input channel will need to be distributed over $n$ photodiodes, each input channel will suffer $1 / n$ power loss. Nevertheless, the milliwatt-level input optical power requirement of the individual enabled wavelength converters and the suppressed background quantum-well absorption in the disabled wavelength converters make large-size crossbars feasible; for example, $n=16$ should be possible. Since these devices avoid photonic interferometric structures, there is no need for precise temperature control or balancing of the individual devices, easing array operation. Because these devices are engineered to operate as isolated circuit elements, the operation speed of the switch elements is independent of the array size $n$, allowing for the operation of large-size arrays at the speed of individual switches in principle.

\section{CONCLUSION}

We have presented a novel $2 \times 2$ low-power compact electrically reconfigurable wavelength-converting crossbar. The crossbar switch demonstrates wavelength conversion across $35 \mathrm{~nm}$ in the $C$-band. Our initial array demonstration leads us to anticipate the feasibility of larger-size wavelength-converting crossbar switches.

\section{ACKNOWLEDGMENT}

The authors would like to thank OEPIC for the epitaxial wafer growth and Melles Griot for the waveguide alignment setup.

\section{REFERENCES}

[1] S. J. B. Yoo, "Wavelength conversion technologies for WDM network applications," J. Lightwave Technol., vol. 14, pp. 955-966, June 1996.

[2] L. A. Coldren, "Widely-tunable chip-scale transmitters and wavelength converters," in Integrated Photonics Research Meeting (IPR), Washington, DC, 2003, Paper IMB1, pp. 6-8.

[3] S. Kodama et al., " $200 \mathrm{Gbit} / \mathrm{s}$ monolithic photodiode-electroabsorption modulator optical gate," in Device Research Conference (DRC), Notre Dame, IN, 2001, Paper VB3, pp. 151-152.

[4] H. V. Demir et al., "Novel scalable wavelength-converting crossbar," in IEEE-OSA Optical Fiber Communications Conf. (OFC), Los Angeles, CA, Feb. 22-27, 2004, Paper FD5.

[5] H. V. Demir et al., "Novel optically-controlled optical switch based on intimate integration of surface-normal photodiode and waveguide electroabsorption modulator for wavelength conversion," in Conf. Lasers and Electro-Optics (LEOS), Tuscon, AZ, 2003, Paper WU1.

[6] V. A. Sabnis et al., "Optically-switched dual-diode electroabsorption modulator," in Integrated Photonics Research Meeting (IPR), Washington, DC, 2003, Paper IMB3, pp. 12-14.

[7] H. V. Demir et al., "Dual-diode quantum-well modulator for C-band wavelength conversion and broadcasting," OSA Opt. Express, vol. 12, no. 2, pp. 310-316, 2004. 